\title{
Star Formation in NGC 4038/4039
}

\author{
Sabine Mengel, Niranjan Thatte, Matthew Lehnert, Reinhard Genzel \\ Max-Planck-Institut für extraterrestrische Physik, Postfach 1312, \\ D-85741 Garching, Germany
}

\begin{abstract}
We performed near infrared integral field spectroscopy of several star clusters and the nuclei of the prototypical merger NGC 4038/39 ("The Antennae"). Near infrared (NIR) images covered both galaxies. The collision of the two gas-rich spiral galaxies has triggered a starburst obvious from a large number of young star clusters. ISO data suggest that the most active star formation occurs in the region where the two galaxy disks overlap. A significant fraction of the total bolometric luminosity of the system is produced there. Since this region is heavily extincted in the optical, the investigation was made in the NIR. Using $\mathrm{Br} \gamma$ emission and $\mathrm{CO}$ absorption features as age indicators, we derive the ages and mass estimates of the star clusters from a comparison with stellar synthesis models. Extinction is calculated from the $\mathrm{Br} \gamma / \mathrm{H} \alpha$ ratio. The young, bright star clusters have ages ranging from 4-12 Myrs, while the nuclear starbursts are much older (50-80 Myrs). The overlap region hosts most of the youngest star clusters below $\sim 8$ Myrs, while the northwestern region is dominated by star clusters in the age range between 8 and 12 Myrs. Several regions, including the northern nucleus, show evidence for sequential star formation on small spatial scales $(<100 \mathrm{pc})$.
\end{abstract}

\section{Introduction, Observations and Results}

Several observations over the last years suggest that galaxy mergers have played an important part in the evolution of galaxies. The Antennae, due to their proximity, offer the possibility to study such and encounter at sub-arcsecond resolution. The merging process is accompanied by a violent burst of star formation. The star clusters seen in The Antennae provide a large number of simple stellar populations, because at their small sizes $\left(R_{\text {eff }}<10 \mathrm{pc}\right)$ all the stars in a clusters have formed during an instantaneous burst. This facilitates the comparison with evolutionary synthesis models. We present the results of this study, performed using NIR imaging spectroscopy and imaging. NIR imaging spectroscopy used MPE-3D at the AAT. Six fields were observed: the nuclei and four star clusters from different parts of the merger, intended to probe different physical environments. The wavelength range was $1.95-2.45 \mu \mathrm{m}$ at a resolution of $\mathrm{R}=\lambda / \Delta \lambda \approx 1000$ and a pixel size of $0^{\prime \prime} 4 /$ pix. NIR images were obtained using SOFI at the NTT. The observed filters included J, H, Ks, $\mathrm{Br} \gamma$ and $\mathrm{CO} \lambda 2.32 \mu \mathrm{m}$ and the corresponding narrow band continuum. The whole merger was covered at a pixel scale of $0.29 /$ pix. $\sim 150$ clusters are detected in 


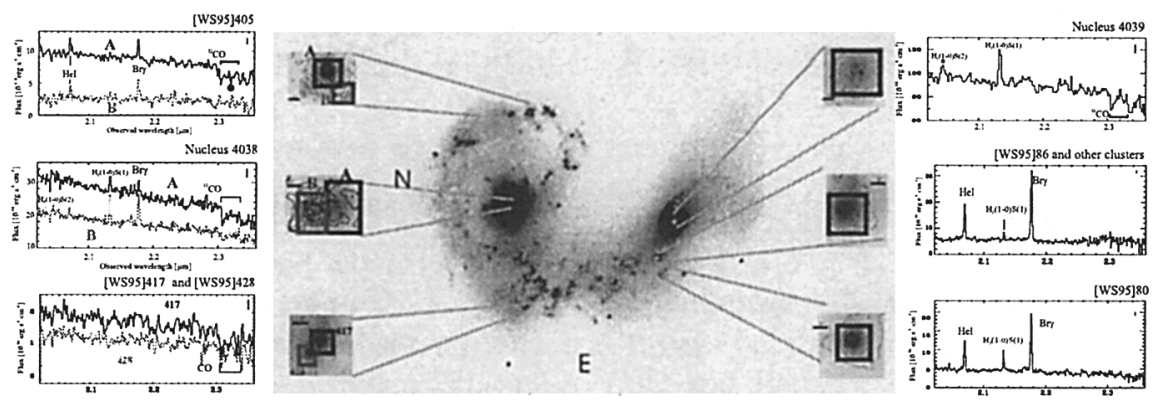

Figure 1. The Ks SOFI image with contour overlays of $\mathrm{Br} \gamma$ emission (light grey, $\mathrm{W}_{\mathrm{Br} \gamma}>30 \AA$, younger than $\sim 7 \mathrm{Myrs}$ ) and $\mathrm{CO}$ absorption (dark grey, $\mathrm{W}_{\mathrm{CO}}>8 \AA$, between 7 and $12 \mathrm{Myrs}$ ) contour overlays. The little images are 3D K-band images with $\mathrm{Br} \gamma$ contours and the spectra extracted from the regions indicated as boxes. Numbers as in Whitmore \& Schweizer (1995).

the Ks image. The equivalent widths in $\mathrm{Br} \gamma$ emission and $\mathrm{CO}$ absorption are determined by photometry. Figure $1^{1}$ shows the images and the spectra. These data, compared with the Starburst99 predictions (Leitherer et al., 1999) suggest that the detected clusters formed less than $\sim 12$ Mrys ago, and that the nuclear starbursts started at least 50 Myrs ago. Older clusters (see Whitmore et al. (1999)) are too faint to be identified here. The extinction, determined from the $\mathrm{Br} \gamma / \mathrm{H} \alpha$, varies from $\mathrm{A}_{V}=0.3$ to $\mathrm{A}_{V} \sim 5$ mag. Extinction corrected photometry in comparison with the expected fluxes at a given cluster age suggests cluster masses between a few $\times 10^{5}$ and a few $\times 10^{6} \mathrm{M}_{\odot}$ for Salpeter IMF between 1 and $100 \mathrm{M}_{\odot}$ and solar metallicity. Spectroscopically determined masses (using ISAAC and UVES, see Mengel et al., 2001b) were as high as these photometric masses or, in some cases, a factor of 2.6 higher.

\section{References}

Leitherer, C., et al. 1999, ApJS, 123, 3

Mengel, S. et al., 2001, to appear in ApJ (March issue)

Mengel et al., 2001b, in preparation

Whitmore, B. \& Schweizer, F. 1995, AJ, 109, 960

Whitmore, B. C. et al., 1999, AJ, 118, 1551

${ }^{1}$ see Mengel et al. 2001a for a more detailed figure and discussion 